Canadian University Music Review

Canadian University Music Review

Revue de musique des universités canadiennes

\title{
La situation de l'orgue au Québec entre 1764 et 1836 telle que présentée par la presse
}

\section{Carole Grégoire}

Volume 10, numéro 1, 1990

URI : https://id.erudit.org/iderudit/1014892ar

DOI : https://doi.org/10.7202/1014892ar

Aller au sommaire du numéro

Éditeur(s)

Canadian University Music Society / Société de musique des universités canadiennes

ISSN

0710-0353 (imprimé)

2291-2436 (numérique)

Découvrir la revue

Citer cet article

Grégoire, C. (1990). La situation de l'orgue au Québec entre 1764 et 1836 telle que présentée par la presse. Canadian University Music Review / Revue de musique des universités canadiennes, 10(1), 1-11.

https://doi.org/10.7202/1014892ar

All Rights Reserved @ Canadian University Music Society / Société de musique des universités canadiennes, 1990
Ce document est protégé par la loi sur le droit d'auteur. L’utilisation des services d'Érudit (y compris la reproduction) est assujettie à sa politique d'utilisation que vous pouvez consulter en ligne.

https://apropos.erudit.org/fr/usagers/politique-dutilisation/ 


\title{
LA SITUATION DE L'ORGUE AU QUÉBEC ENTRE 1764 ET 1836 TELLE QUE PRÉSENTÉE PAR LA PRESSE
}

\author{
Carole Grégoire
}

L'histoire de l'orgue au Québec débute dès l'époque de la Nouvelle-France. D'après le Journal des Jésuites, déjà en 1661, la chapelle des Jésuites possède son instrument. Deux ans plus tard, Mgr François de Laval dote à son tour l'église paroissiale de Québec d'un orgue à tuyaux. Quant à Montréal, il faut attendre la fin du 17e siècle pour qu'un orgue soit installé à l'église Notre-Dame.

Avec l'avènement du régime anglais, il est attesté que l'orgue trouve place dans les églises de différentes confessions. Il suffit de mentionner les cathédrales catholique et anglicane de Québec au début du 19e siècle, qui reçoivent chacune un orgue du facteur Elliott de Londres, faute de trouver au pays des facteurs professionnels.

Malgré les preuves fournies sur l'implantation de l'orgue au Québec au cours des premiers siècles de son histoire, il faut reconnaître que les ouvrages sont peu éloquents sur le sujet. La cause en est attribuable en partie à la pénurie des sources que sont principalement les archives et les mémoires, mais également à l'incapacité de recourir à la presse comme source principale d'informations.

La presse nous paraissant être la source la plus régulière de renseignements sur le sujet, nous avons fait un relevé systématique des informations traitant de l'orgue contenues dans la presse québécoise entre 1764, date d'apparition de cette dernière, et 1836, année correspondant à l'arrivée à Montréal d'un important facteur d'orgues, Samuel Russell Warren. Ce relevé de 400 informations a permis d'établir une chronologie qui apportera une certaine connaissance de l'histoire de l'orgue au Québec ${ }^{1}$. Quatre aspects ressortent de ces

1 Les informations ont été puisées dans la banque de données de l'équipe de recherche «Histoire de la musique au Québec (1764-1918) d'après la presse québécoise de l'époque », dirigée par Lucien Poirier et Juliette Bourassa-Trépanier. 
informations de la presse : l'organologie, la facture, l'usage des instruments et les organistes. Nous ferons le point sur les instruments et leurs facteurs.

\section{Les instruments}

La presse présente trois catégories d'instruments : les orgues à clavier(s), les orgues à cyclindre(s) et les orgues à clavier(s) et à cylindre(s). Nous tenterons de définir quelles sont les caractéristiques de chaque catégorie.

Le corpus des données relatives aux orgues à clavier(s) comporte une trentaine d'éléments dont la majorité figure après 1822 . Leur étude révèle l'existence d'instruments à trois, deux et surtout un clavier. Les plus considérables sont désignés orgues d'église, tandis que les autres reçoivent généralement l'appellation d'orgues de chambre. Dès 1764, La Gazette de Québec décrit un de ces instruments :

Une belle orgue à cinq clefs, qui conviendroit à une petite église ou à une chambre $^{2}$.

L'étendue des claviers qui, on le sait, conditionne le nombre de tuyaux, fait assez souvent l'objet de précisions intéressantes. Le nombre habituel de touches est de 61, ce qui correspond à un clavier de cinq octaves. Cependant, les facteurs Jacotel \& Fay construisent au moins un orgue dérogeant à l'usage, tel que le rapporte un article de La Minerve de 1831:

MM. Jacotel \& Fay viennent de compléter un autre grand orgue d'église, de 6 octaves, qu'ils offrent en vente à leur atelier au côteau Barron, près de cette ville, $[\ldots]^{3}$.

Une autre particularité de l'instrument concerne sa composition qui offre une ressemblance frappante avec les instruments anglais. L'orgue à un clavier, utilisé au Québec à la fin du 18e siècle et au début du 19e, comprend entre cinq et huit jeux, dont les plus fréquents sont :

\section{Diapason ouvert \\ Diapason fermé \\ Principal \\ Flûte \\ Cornet \\ Sesquialtera}

2 The Quebec Gazette/La Gazette de Québec, 17 (11 oct. 1764) : 3.

3 La Minerve, $5 / 39$ (27 juin 1831) : 3. 
Malgré leur rareté, certains instruments de plus grandes dimensions conservent les éléments de base de l'instrument à un clavier. La description d'un orgue à deux claviers fabriqué au début de l'année 1837 par Samuel Russell Warren et George Mead pour l'église de Berthier en témoigne :

Le jeu d'écho surtout a un effet admirable. Il est de trois octaves et de quatre régistes : le hautbois, le principal, le diapason fermé et ouvert. Le grand-orgue est de sept régistes, et de cinq octaves, et douze touches-pédales dans les basses $^{4}$.

La description complète de l'orgue de Berthier nous permet de constater que les facteurs locaux ont dû adapter leurs instruments pour répondre à des besoins particuliers. Ainsi, en raison du climat, on a parfois retranché des jeux d'anches :

Nous félicitons le facteur de n'avoir mis dans son orgue qu'un seul jeu d'anches. Les jeux de fonds conviennent infiniment mieux à notre climat changeant, car ils sont moins sujets à se déranger. Les orgues qui nous viennent d'Angleterre ont ordinairement 4 à 5 jeux d'anches $[\ldots]^{5}$.

L'article fournit également des précisions intéressantes entourant l'importation d'instruments anglais au Québec. C'est le cas de l'orgue à trois claviers de l'église épiscopale protestante de Montréal, seul exemple détaillé d'un instrument de cette dimension qui, quoique plus élaboré, possède aussi les éléments caractéristiques de l'instrument à un clavier :

$\begin{array}{lll}\text { Grand-orgue } & \text { Récit } & \text { Positif } \\ \text { 3 diapasons } & \text { 2 diapasons } & \text { 2 diapasons } \\ \text { Principal } & \text { Principal } & \text { Principal } \\ \text { Twelfth } & \text { Trumpet } & \text { Flute } \\ \text { Fifteenth } & \text { Hautboy } & \text { Fifteenth } \\ \text { Sesquialtera } & & \\ \text { Cornet } & & \\ \text { Mixture } & & \\ \text { Trumpet } & & \\ \text { Clarion } & & \end{array}$

Tous ces instruments ne semblent pas avoir eu de pédalier. Dans le cas contraire, la presse en fait état.

4 La Minerve, 10/100 (23 janv. 1837) : 3.

5 Idem. 
Le grand orgue est de sept régistres et de cinq octaves et douze touchespédales dans les basses ${ }^{6}$.

On peut toutefois se demander si, en l'absence de jeux propres à la pédale, des touches dans les basses du clavier principal n'auraient pas été ajoutées. Vraisemblablement, on peut y voir une autre manifestation de l'influence anglaise; car l'orgue anglais doit attendre la fin du $18 \mathrm{e}$ siècle pour avoir un pédalier et le 19e, pour des registres de pédales (Owen 1979: 38).

En ce qui a trait aux orgues à cylindre(s), on leur a accordé jusqu'ici peu d'importance. La presse en fait cependant grand état : plus de soixante-douze instruments, munis de un à six cylindres sont présentés, la majorité après 1810.

Dans un pays où les interprètes étaient rares, on a pu chercher à utiliser des instruments capables de reproduire mécaniquement le jeu d'un musicien sur son instrument, par le biais de cylindre(s).

Le principe mécanique général de ces orgues est le suivant : une manivelle fait tourner une vis sans fin et un excentrique. La vis sans fin fait tourner à son tour le cylindre alors que l'excentrique fait aller et venir le soufflet qui alimente l'instrument en vent. Le cylindre constitue l'élément le plus important de l'instrument, car c'est sur ce dernier que sont notées les différentes pièces musicales. Un cylindre contient habituellement huit ou dix airs.

Tous les orgues à cylindre(s) sont fabriqués sur le même principe et ne diffèrent que par leur dimension. La taille des instruments varie en fonction du nombre de cylindre(s) que possède un instrument.

Les instruments les plus couramment utilisés sont ceux à trois ou quatre cylindre(s). Nous avons retracé au Québec un de ces instruments, si populaire à l'époque, en l'église anglicane St-Thomas à Rougemont. L'instrument, fabriqué en 1844 par Joseph Walker, possède trois cylindres sur chacun desquels sont notées dix pièces, ainsi que trois jeux : un diapason ouvert, une dulciana et un principal.

Le répertoire joué par cet instrument comprend de la musique religieuse, puisqu'il a trouvé place dans une église. Il en est autrement des orgues destinés à l'usage domestique. Le répertoire de ces instruments était composé

Idem. 
de pièces connues et populaires. Les mentions «airs choisis», "nouvelles pièces», «à la mode» sont fréquentes tel que l'illustre l'extrait suivant :

[...] un élégant orgue à tuyeau jouant 50 des airs les plus choisis $[\ldots]^{7}$.

De plus, ces instruments possèdent des jeux d'accompagnement comprenant des percussions; les plus couramment cités sont le triangle, le tambour et le carillon.

Toujours en rapport avec les orgues à cylindre(s), cinq orgues à clavier(s) et à cylindre(s) ont également été mis en vente entre 1811 et 1834 . Le principe mécanique de ces instruments est celui de l'orgue à cylindre(s) jumelé à celui de l'orgue à clavier(s). Ce qui en fait des instruments polyvalents, puisqu'en l'absence d'un organiste, on peut quand même exécuter un répertoire courant, et dont la qualité semble satisfaire les critères du temps. Un article du Spectateur canadien nous renseigne sur les qualités musicales d'un de ces instruments fabriqué par Jacotel \& Fay:

[...] cet instrument qui a eu l'approbation des meilleurs connaisseurs de Montréal est d'une harmonie supérieure, d'un accord parfait et d'un effet extraordinaire pour sa dimension ${ }^{8}$.

Deux des cinq instruments répertoriés possèdent respectivement trois et quatre cylindres. On peut donc supposer que ces instruments étaient de dimensions semblables à celles des orgues à cylindre(s) les plus fréquemment utilisés.

\section{Les facteurs}

Mais tous ces instruments, par qui étaient-ils fabriqués, entretenus et accordés? De la fin du 18e siècle au début du 19e, nous l'avons dit, les orgues du Québec proviennent généralement d'Europe, plus précisément d'Angleterre. La place importante attribuée à l'instrument par les différentes confessions contribue à augmenter la demande des églises et des particuliers. Pendant que s'accentue ce mouvement, on assiste, dès les années 1820 , aux premières manifestations d'une facture locale de l'orgue au Québec. Afin de promouvoir l'implantation, les artisans doivent d'abord démontrer que les produits canadiens sont d'aussi bonne qualité que les instruments importés. C'est ce que tente Auguste Fay de Montréal en 1836, comme le précise cet entrefilet:

7 Le Spectateur canadien, 4/3 (10 juin 1816) : 3.

8 Le Spectateur canadien, 13/30 (3 septembre 1825) : 3. 
Il a déjà exécuté plusieurs orgues et nommément celles de Ste-Thérèse et de Ste-Anne, qui ont fait l'admiration des connaisseurs. Elles n'en cèdent à rien à celles qui sont importées, quant à l'harmonie et à la solidité 9 .

Différentes raisons expliquent l'apparition et le progrès de la facture d'orgue au Québec. D'abord, les coûts de l'importation, estimés à quelque $25 \%$ de plus que les instruments locaux, subissent une nouvelle majoration en $1825^{10}$. À des raisons d'ordre économique s'ajoute l'exigence de réparer, voire remplacer l'orgue en cas de sinistre; phénomène assez fréquent à l'époque. Par ailleurs, le climat canadien est trop rigoureux pour plusieurs des instruments européens. Finalement, l'arrivée d'immigrants, parmi lesquels se trouvent d'habiles facteurs, contribue au développement de la facture d'orgue dans la province (Kallmann 1960 : 115-6).

La presse de 1764 à 1836 permet d'identifier vingt-six firmes ou personnes différentes s'occupant de facture d'orgue : ce nombre comprend les amateurs et les professionnels. Les mentions ne sont toutefois que sporadiques. Malgré tout, il s'agit d'une amélioration sur l'époque précédente, celle de la fin du $18 \mathrm{e}$ siècle, où la presse ne signale aucun nom. Selon ces données, et à défaut d'autres témoignages connus, la facture d'orgue au Québec remonterait au début du 19e siècle, mis à part un instrument fabriqué en 1723 par Paul Jourdain dit Labrosse pour la cathédrale de Québec (Bouchard 1983 : 773).

Les annonces publiées permettent assez facilement d'identifier les facteurs spécialisés. Le facteur spécialisé, nous le présentons comme celui qui conçoit et réalise l'instrument; il est facteur d'orgue par profession, tel que le montre cette annonce :

Aux amateurs de musique - M. Samuel Russell Warren, facteur d'orgues, ci-devant de Boston chez Appleton, informe respectueusement le public que le 22e jour d'octobre dernier, il est entré en société avec Mr. George Mead, ci-devant de la Maison Mead, Mott and Cie, facteurs de pianos fortes, aux fins de confectionner les orgues d'églises, de toute dénomination, sous les noms et raison de Mead \& Warren ${ }^{11}$.

Le tableau suivant dresse la liste chronologique des facteurs spécialisés en

$9 \quad$ La Minerve, 10/14 (31 mars 1836) : 3 .

10 Canadien Courant and Montreal Advertiser, 19/3 (21 mai 1825) : 2.

11 La Minerve, 10/99 (23 janvier 1837) : 3. 
TABLEAU 1

\begin{tabular}{lll}
\hline Raison sociale & Années de mention & Lieu d'activité \\
\hline Jean-Baptiste Jacotel & 1821,1823 & Montréal \\
Auguste Malard & 1821,1826 & Montréal, Québec \\
$\begin{array}{l}\text { J.B. Jacotel \& } \\
\quad \text { Auguste Fay }\end{array}$ & $1825,1830,1831$ & Montréal \\
$\begin{array}{l}\text { George Zingraff \& } \\
\text { Joseph Bourdon }\end{array}$ & 1825 & Montréal \\
Joseph Bourdon & 1825,1827 & Montréal \\
George Zingraff & 1830 & Montréal \\
Auguste Fay \& & 1832 & Montréal \\
$\quad$ M. Jacotel, fils & 1835 & \\
R. Mead \& fils & 1836 & Montréal \\
Joseph Jacotel, fils & 1836,1837 & Montréal \\
John W. Herbert \& & & Montréal \\
$\quad$ William Dennis & 1836 & Montréal \\
Auguste Fay & 1837 & Montréal \\
Mead \& Warren & 1837 & Montréal \\
Samuel Russell Warren & &
\end{tabular}

faisant mention de leur nom, de leurs années de mention et de leur lieu d'activité.

Quatre des dix facteurs sont originaires de la France: Jean-Baptiste Jacotel, Auguste Fay, Auguste Malard et George Zingraff; un autre vient des ÉtatsUnis, Samuel Russell Warren. Helmut Kallmann (1960) a donc raison d'affirmer que l'arrivée d'immigrants, notamment la venue de Samuel Russell Warren, a favorisé le développement de la facture d'orgue au Québec. Fait étonnant, on ne mentionne personne venu de l'Angleterre.

Le métier de facteur d'orgue suppose, chez le facteur, un grand nombre de connaissances; la presse ne nous permet toutefois pas d'en vérifier l'étendue. Elle nous autorise cependant à définir les principales activités du facteur. D'une façon générale, il s'occupait parallèlement de facture, d'accord et de réparation. Une annonce publiée par Joseph Jacotel en 1836 en témoigne : 
M. Joseph Jacotelle, fils, successeur de feu M. J.B. Jacotelle, facteur d'orgues, informe les marguilliers et le public en général qu'il continuera toujours sa manufacture d'ORGUES D'ÉGLISE et de tout autre genre. Il se transportera dans les paroisses où l'on voudra bien l'appeler pour réparer, accorder, raccommoder les orgues ${ }^{12}$.

Les instruments que construisent, réparent, accordent ces facteurs sont des orgues d'église, des orgues de chambre, à cylindre(s) et/ou à clavier(s). La presse ne dit pas si les orgues étaient entièrement fabriqués ici. Un article de Charles Chapais, datant de 1937, nous invite à penser que certaines parties des instruments pouvaient être importées :

Avant lui [Joseph Casavant], les orgues de nos églises étaient importés d'Angleterre, d'Allemagne ou de France. De son temps, l'on importait de ces pays des tuyaux d'orgues pour compléter des instruments construits au pays; mais Joseph Casavant est le premier canadien-français qui ait offert à sa clientèle un produit exclusivement canadien, à l'exception, toutefois, de quelques instruments construits au temps de Mgr Laval par un ecclésiastique du pays (Chapais $1937: 547$ ).

À l'appui des dires de Chapais, l'assemblage d'instruments importés était pratiqué au Québec. Seule la firme J.W. Herbert \& Cie en fait la publicité.

Comme nous le mentionnions précédemment, les orgues à cylindre(s) étaient bien en vogue à l'époque. En 1825, la firme Jean-Baptiste Jacotel \& Auguste Fay annonce que les facteurs «excellent surtout dans la nototechnie ou art de noter sur cylindre ${ }^{13}$. Ils sont eux aussi les seuls à faire de la publicité concernant ce type d'activité.

Bien que la facture d'orgue soit une activité spécialisée, elle a également intéressé des personnes exerçant d'autres professions mais cultivant cet art par nécessité. Une mention, au sujet d'un facteur non-spécialisé, illustre bien la polyvalence de certains d'entre eux.

Le soussigné prend la liberté de remercier les amis et le public en général des faveurs qu'il a éprouvées de leur part, et les sollicite humblement de l'encourager dans les ouvrages suivants qui consistent à mettre d'accord et à réparer de la manière la plus expéditive des orgues à doigts et à barils, harpes, piano fortes, et tout autre instrument de musique, ainsi qu'à graver des chiffres, à faire des gravures pour des cartes et des billets, à faire les ouvrages les plus

L'Ami du peuple, de l'ordre et des lois, 4/77 (13 avril 1836) : 307.

13

Le Spectateur canadien, 13/30 (3 septembre 1825) : 3 . 
TABLEAU 2

\begin{tabular}{|c|c|c|c|}
\hline Raison socale & Années & Profession & Lieu \\
\hline Thomas Fitzer & 1805 & «Mechanic» & Montréal \\
\hline M. Dyke & 1813 & indéterminé & $\begin{array}{l}\text { Québec (fév.) } \\
\text { Montréal } \\
\text { (juin) }\end{array}$ \\
\hline Thomas Marsden & 1816,1818 & professeur & Québec \\
\hline Frederick Hund & $1816,1817,1818$ & facteur de piano & $\begin{array}{l}\text { Québec } \\
(1816-1817) ; \\
\text { Montréal } \\
(1817-1818)\end{array}$ \\
\hline Samuel Frost & 1818 & ébéniste & Montréal \\
\hline Frederick Hoffmaster & 1819 & indéterminé & $\begin{array}{l}\text { Québec } \\
\text { (juin); } \\
\text { Montréal } \\
\text { (juillet) }\end{array}$ \\
\hline Hund \& Seebold & 1820,1821 & facteurs de piano & $\begin{array}{l}\text { Québec } \\
\text { (1820); } \\
\text { Montréal } \\
(1821)\end{array}$ \\
\hline W.W. Andrews & 1824 & $\begin{array}{l}\text { professeur de } \\
\text { musique et de } \\
\text { dessin }\end{array}$ & Montréal \\
\hline Thomas Baillargé & 1829 & architecte & Québec \\
\hline W. Warren & 1829 & $\begin{array}{l}\text { professeur de } \\
\text { musique }\end{array}$ & Montréal \\
\hline J. Wood & 1832 & horloger & Montréal \\
\hline John Thomas & 1835 & facteur de piano & Montréal \\
\hline George Pringle & 1836 & horloger & Montréal \\
\hline
\end{tabular}

élégants en cheveux, et à réparer et à perfectionner des machines électriques. Tout ordre qui sera envoyé chez Mr. Gibb, rue Notre-Dame, sera reçu avec reconnaissance, et exécuté avec ponctualité, par Thomas Fitzer ${ }^{14}$.

${ }^{14}$ La Gazette de Montréal/The Montreal Gazette, 538 (2 déc. 1805) : 3. 
$\mathrm{Au}$ Québec, treize personnes appartenant à cette catégorie des facteurs amateurs ont été identifiées. Leurs professions principales les rangent parmi les professeurs (3), les facteurs de piano (3), les horlogers (2), tandis que trois autres sont respectivement ébéniste, architecte et mécanicien. Voici la liste de ces personnages, les années durant lesquelles ils ont été mentionnés, leur profession ainsi que leur lieu d'activité : tableau 2.

Contrairement aux facteurs spécialisés qui sont concentrés presque exclusivement à Montréal, sept exercent leur métier à Montréal, quatre à Québec et à Montréal, et deux à Québec.

Devant le manque de spécialistes en la matière, ces personnes ont vraisemblablement dû s'improviser pour répondre aux besoins du milieu. À Québec, par exemple, à l'exception de Auguste Malard en 1826, on ne mentionne aucun facteur spécialisé. On note toutefois la présence de facteurs non spécialisés en $1813,1816,1818,1819,1820$ et 1829.

En ce qui a trait à leurs activités, les facteurs amateurs s'occupent majoritairement d'accord et de réparation; seuls les horlogers Wood et Pringle parlent de facture. Thomas Baillargé est une exception, il confectionne, en 1829, le buffet de l'orgue de la cathédrale de Québec.

En conclusion, l'histoire de l'orgue au Québec est à faire et la presse apporte un éclairage nouveau. Grâce à la presse, on peut à la fois évaluer l'importance de l'instrument à l'époque et l'usage que l'on faisait de certains instruments maintenant laissés de côté, comme l'orgue à cylindre(s). Par ailleurs, de nombreux facteurs seraient tombés dans l'oubli si la presse ne nous avait transmis leurs noms et un certain aperçu de leurs réalisations. La compilation de ces informations nous reporte à une époque bien différente de la nôtre, mais nous permet d'affirmer que l'orgue s'est implanté au Québec pour y demeurer encore bien vivant.

\section{RÉFÉRENCES}

BOUCHARD, Antoine

1983 : «Orgue-Facture», Encyclopédie de la musique au Canada, Montréal : Fidès.

CHAPAIS, Charles

1937 : «La construction des orgues par les Canadiens-français», Congrès de la langue française du Canada, 2. 


\section{KALLMANN, Helmut}

1960: A History of Music in Canada 1534-1914. Toronto: University of Toronto Press.

OWEN, Barbara

1979 : The Organ in New England. Raleigh: The Sunbury Press. 